XIIII.

Aus der psychiatrischen Unicersitätsklinik zu lena

(Direktor: Prof. Dr. Reroer).

\title{
Ueber intravenöse Injektionen hoher Dosen von Natrium nucleinicum beim Menschen.
}

\author{
Yon \\ Dr. W. Jacobi, \\ Assistenzarzt. \\ Mit + Knrren im Text.)
}

Die Tatsache, dass bei der progressiven Paralyse Remissionen im Zusammenhang mit fieberhaften Erkrankungen und im Anschluss an langdauernde profuse Eiterungen beobachtet werden, hatte seiner Zeit dazu geführt, der Nukleinsüure lingang in die Behandlungsmethoden der progressiven Paralyse zu rerschalfen. Durch Hyperleukozytose und Hyperthermie wollte man die Oxydationsforgänge im Körper heben und auf diese Weise in diesem entstandene giftige Stoffwechselprodukte des Nervengewebes beseitigen. Die Lehre Il etschnik off's, dass den Leukozyten bei der Eliminierung toxischer Stoffe aus dem Körper eine grosse Bedeutung zukäme und dass das hı̣perleukozytäre Blut stark bakterizide Wirkung besässe, spielte bei diesen Erwägungen wohl eine ausschlaggebende Rolle.

Parlavecchiol) hatte dann daraul hingewiesen, dass die Nukleinsäure durch Steigerung der dbwehrkräfte des Organismus, d. h. durch Erhöhung des Alexin- und (1psoningehaltes und dadurch, dass sie dem Serum ein Agglutinationsvermögen verleiht, wirkt, und 'l'schernoruzki2, hatte festgestellt, dass Einführung dieses Stoffes in den Organismus einen deutlichen Einfluss auf seine fermentative 'Tätigkeit, besonders auf dir des Gehirns, ausübt. Paoli und ( $a$ alisti $^{3}$ ) hatten unter Beweis gestellt, dass die Nukleinsäureinjektion wie eine Infektion wirkt. also albwehrkräfte und Immunisierungsprozesse im Körper wachruft.

1) G. Parlarecchio, Ceber die immunisierende Wirkung der Nukleinsäure. Arch. f. klin. Chir. (Langenbeck). 1909. Bd. 90. S. 202.

2) N. Tschernoruzki, Ceber die.Wirkung der Nukleinsäure auf die fermentativen Prozesse im tierischen Organismus. Biochem. Zeitschr. 1911. Bd. 36. S.363.

3) Erasmo de Paoli und Ant. Calisti, Beobachtungen und experimentelle Untersuchungen über den Wert der Injektion des nukleinsauren Natriums bei der Prophylaxe der operativen Infektion des Peritoneums. Mitt. a. d. Grenzgeb. d. Med. u. Chir. 1912. Bd. 24. H. 2. 
Donath, Fischer, Jurmann und Tsiminakis hatten über Erfolge der Nukleinsäuretherapie bei progressiver Paralyse berichtet, de Block, Hüssels, Jolovicz und Klieneberger über Misserfolge ${ }^{1}$ ).

Unangenehm bei der Nukleinsäuretherapie war die Erfahrungstatsache, dass die subkutanen Injektionen meist schmerzhaft empfunden wurden und häufig zu Infiltratẹn, ja sogar zu $\Lambda$ bszessen führten. Ich ïberlegte, ob ich diese Missstände nicht durch intravenöse Darreichung des Mittels boheben konnte, wie sie $z$. $\beta$, von Buia bei der Nukleinsäurebehandlung der Paralysis agitans empfohlen war ${ }^{2}$ ). Dieser hatte alle Tage $0,02-0,5 \mathrm{~g}$ Natrium nucleinicum in $\bar{\jmath}-10$ proz. wässeriger Lösung teils subkutan, teils intravenös injiziert $\left.{ }^{3}\right)$. Massgebend für mein V'orgehen war die Fragestellung, ob ich bei intravenöser Applikation des Mittels voraussichtlich mit unangenehmen Zwischenfällen zu rechnen hatte.

Nach 'l schernoruzkit) wurde hefenukleinsaures Natron Merck von Hunden bei langandauernder intravenöser Verabreichung grosser Dosen (bis $1,5 \mathrm{~g}$ auf $1 \mathrm{~kg}$ Körpergewicht) gut vertragen, und Ewald ${ }^{5}$ ), der Hunden in wenigen Tagen Abstand 4-5 g Hefenukleinsäure in $100-150 \mathrm{ccm}$ Wasser gelöst intravenös beibrachte, sah keine stärkere toxische Wirkung. Zu bedenken war allerdings, dass der Hund ja ein ziemlich unempfindliches Tier ist, ein Tier, bei dem man $z$. B. auch mit den grössten Dosen sensibilisierender Substanzen bei der Reinjektion keinen anaphylaktischen Tod erzielen kann ${ }^{8}$. Anaphylaktische Forgänge hatte ich $\mathrm{m}$. E. bei dem von mir geplanten Vorgehen nicht zu befürchten, da Nukleinsäure, die ja aus Nukleoproteiden durch völlige Abspaltung der Eiweissstoffe entstehi, keine Eiweissreaktion gibt. So vermochten auch $\Lambda$ bderhalden und Kashiwado ${ }^{7}$ ) mit den reinen Nukleinsäuren in keinem Fall Anaphylaxie zu erzeugen. Dic bei der Spaltung entstehenden Nuklein- und Pyrimidinbasen konnien $m$. E. auch nicht zu unangenehmen Zwischenfällen führen, da diese Stoffe ja zu den normalerweise im Harn vorkommenden Stoffwechselprodukten gehören.

Für die intravenöse Einverleibung des Mittels entschloss ich mich nach theoretischer Ausschliessung schädlicher Nebenwirkungen hauptsächlich aus dem Grunde, weil Nukleinsäure nach den Beobachtungen Tschernoruzki's bei dieser Art der Darreichung die grösste Wirkung auf die fermentative Funktion des Organismus ausübt. Ausserdem lag

1) N. Schacherl, Zum gegenwärtigen Stand der Therapie der nervösen Spätlues. Jahrb. f. Psych. u. Neurol. 1918. Bd. 38. H. 2 u. 3.

2) Buia, Spitalul. 1914. No. 6. - Semaine méd. 1914. No. 13. p. 1555.

3) Vgl. E. Merck's Jahresber. 1914. Jahrg. 28.

4) Vgl. Biochem. Zeitschr. 1911. Bd. 36. S. 363.

5) G. Ewald, Ueber intravenöse Verabreichung von Nukleinsäure und ibren Abbauprodukten beim Hund. Zeitschr. f. exper. Pathol. u. Therap. 1913. Bd. 12.

6) Vgl. "Die Anaphylaxio" von E. Friedberger in: Fortschritte d. deutschen Klinik, herausgegeben von Leyden u. Klemperer. 1911.

7) E. Abderbalden u. T. Kashiwado, Studien über die Kerne der 'Thymusdrüse und Anaphylazieversuche mit Kernsubstanzen (Nukleoproteiden, Nukleinen und Nukleinsäuren). Zeitschr. f. physiol. Chemie. 1912. Bd. 81. S. 285. 
mir daran, festzustellen, ob vermittels der durch Nukleinsäure hervorgerufenen Hyperleakozytose ein Transport des vorher durch intramuskuläre Applikation im Körper deponierten Quecksilbers an den Ort der luetischen Schädigung bewerkstelligt werden könnte, und ob Neosalvarsan, auf der Höhe des Fiebers injiziert, im Liquor nachzuweisen sei. Es sei gleich vorausgenommen, dass es uns nicht gelang, Quecksilber, Arsen oder Jod, das wir in 50 proz. Jodnatriumlösung mittels $20 \mathrm{~cm}$ Spritze auf dem Gipfel des Temperaturanstieges in cinzelnen Fällen einspritzten, in der Lumbalflüssigkeit festzustellen.

Ich injizierte die Nukleinsäure nach dem Prinzip der aktiven Immunisation in allmählich sich steigernden Dosen in etwa achttägigen 7wischenräumen. Als Anfangsdosis gab ich $1 \mathrm{~g}$ Natrium nucleinicum in 10 proz. physiologischer Kochsalzlösung: als Höchstdosis einmal $3 \mathrm{~g}$.

Aus den beigegebenen Kurven erhellt, wie verschieden die einzelnen Kranken reagierten. Bei sämtlichen rier Patientinnen handelte es sich um einwandfreie Fälle von Paralysis progressiva, nur unterschieden durch den Grad der Erkrankung. Gerade die beiden Kranken Gr. und Wi. (Kurve 1 und 2). die auf Nukleinsänreinjektionen so ausserordentlich schwach reagierten. unterschieden sich nach dieser Richtung. Dic Erstere stellte einen ganz frischen Fall dar, der draussen in Leben durch urteilsschwache Eigentumsvergehen aufgefallen war und bei des I ufnahme keinerlei krankhaften Reflexbefund zeigte. bei der die Diagnose lediglich durch Blut- und Liquorbefund gesichert wurde. Anders die Patientin Wi.. die ém trosloses Bild des Endzustandes der Erkrankung darbot. in ihrem Kastenbell in sich zusammengekrochen hockte, vollkommen rertiert war. liut und l'rin unter sich liess und damit schmierte. Fräulein $\mathrm{Ab}$. (Kurve 3 . eine klassisch euphorische Paralyse, war seit etwa einem Jahr psychisch aulgefallen. während Frau Se. Kurve 4), eine 29 jährige Kehrfrau. erst seit einem Monat eine Veränderung ihres Wesens geboten hatte.

Die Kurven zejge山. wie bei intravenöser Einführung des Mittels teilweise ausserordentlich starke, of tagelang anhaltende, in ihrer Höhe zuweilen stündlich lebhaft schwankende Fieberreaktionen hervorgerufen werden. Sie beweisen aber gleichzeitig. dass auch bei dieser Art der Darreichung cine (iewribnung des (Urganismus au das Mittel eintritt ${ }^{\mathbf{l}}$ ). Dies zeigt eine gewisse Beziehung des Mittels zu den immunisicrenden eiweissartigen Substanzen. worauf $u$. a. schon Enge ${ }^{2}$ ingewiesen hatte. Eine Analogie der Wirkung des Yittels zur Infektion ergibt sich zudem m. E. auch aus der mit der Steigerung der Temperatur einhergehenden Hyperleukozytose, der Icbhaftigkeit fermentativer Prozesse und des Stoffwechsels. Die Leukozrtenzahl nahm oft exquisit hohe Werte bis zu $72000 \mathrm{im}$ Kubikmillimeter an und war auch in der zwischen den einzelnen Injektionen liegenden Spanne Zeit zur Norm erhöht. Auffallend war, dass unsere Kranken besonders heftige Reaktionen bei der Reinjektion des in seiner Dosis nicht gesteigerten Mittels in einer Zeit

1) Vgl. J. Donath, Berl. klin. Wochenschr. 1910. S. 2343.

2) Zeitscbr. f. d. ges. Neurol. u. Psych. 1912. Bd. 4. H. 6. 
Kurve 1. (Gr.)
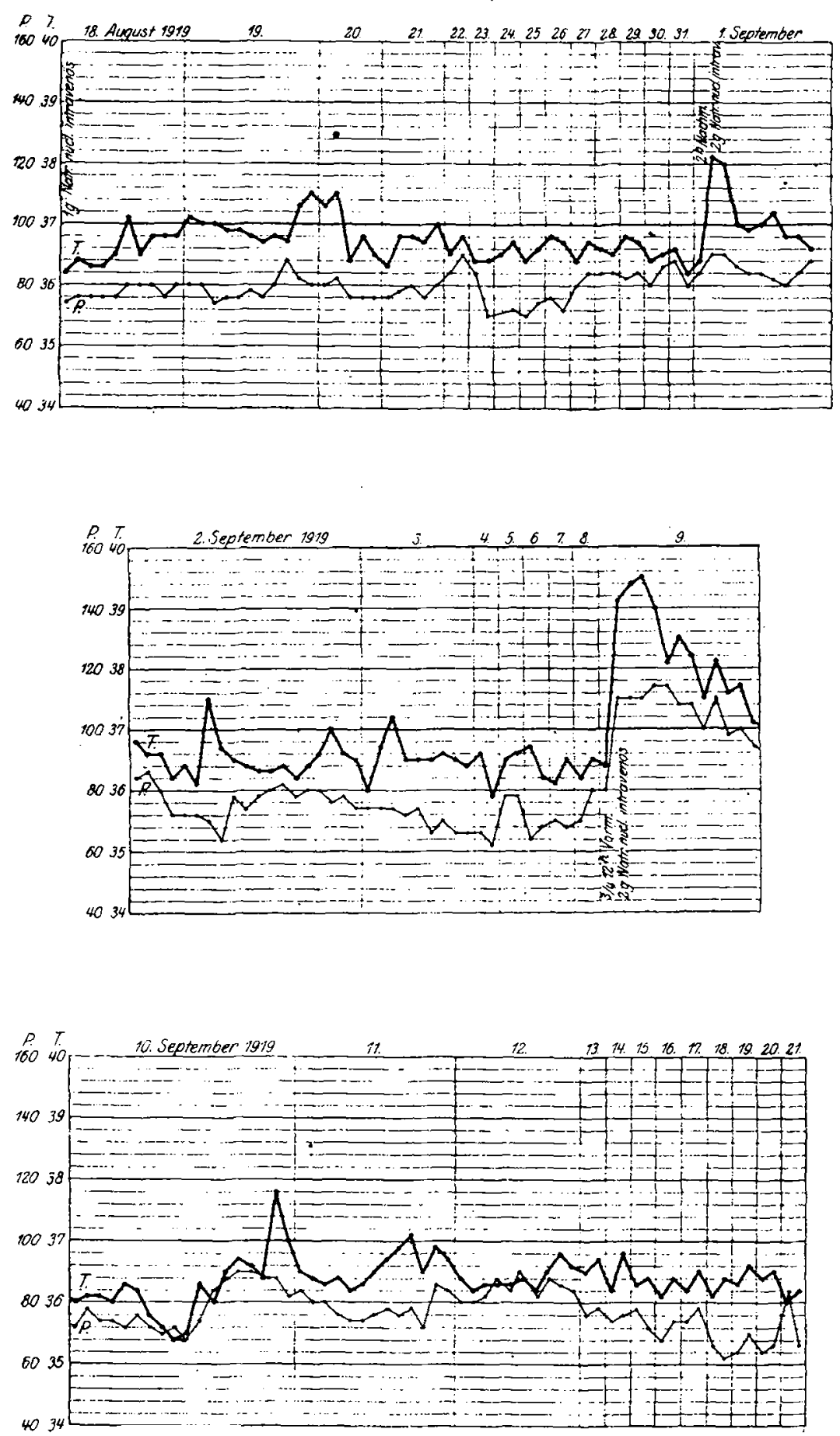
Kurvo 2. (Wi.)
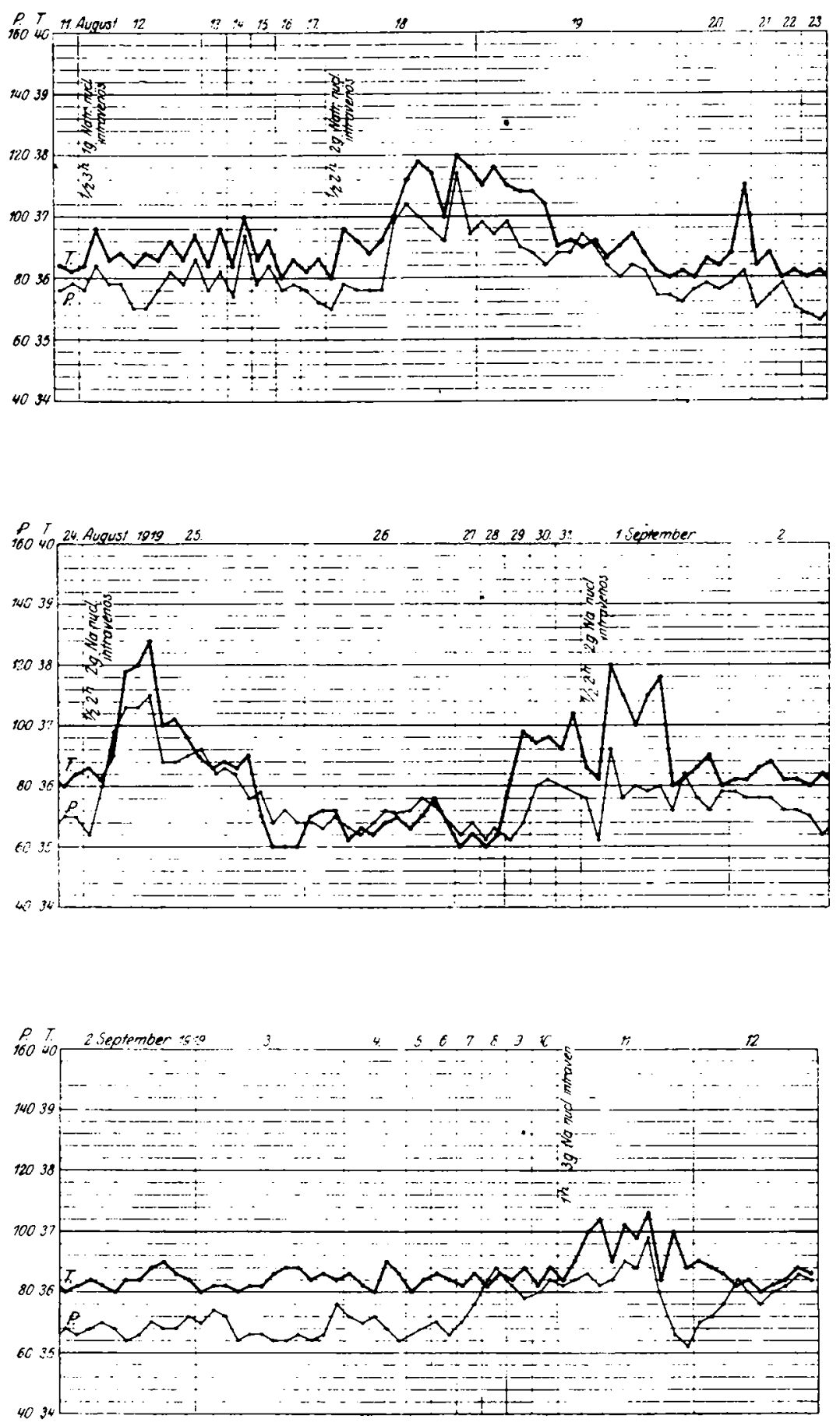
Kurve 3. (Ab.)
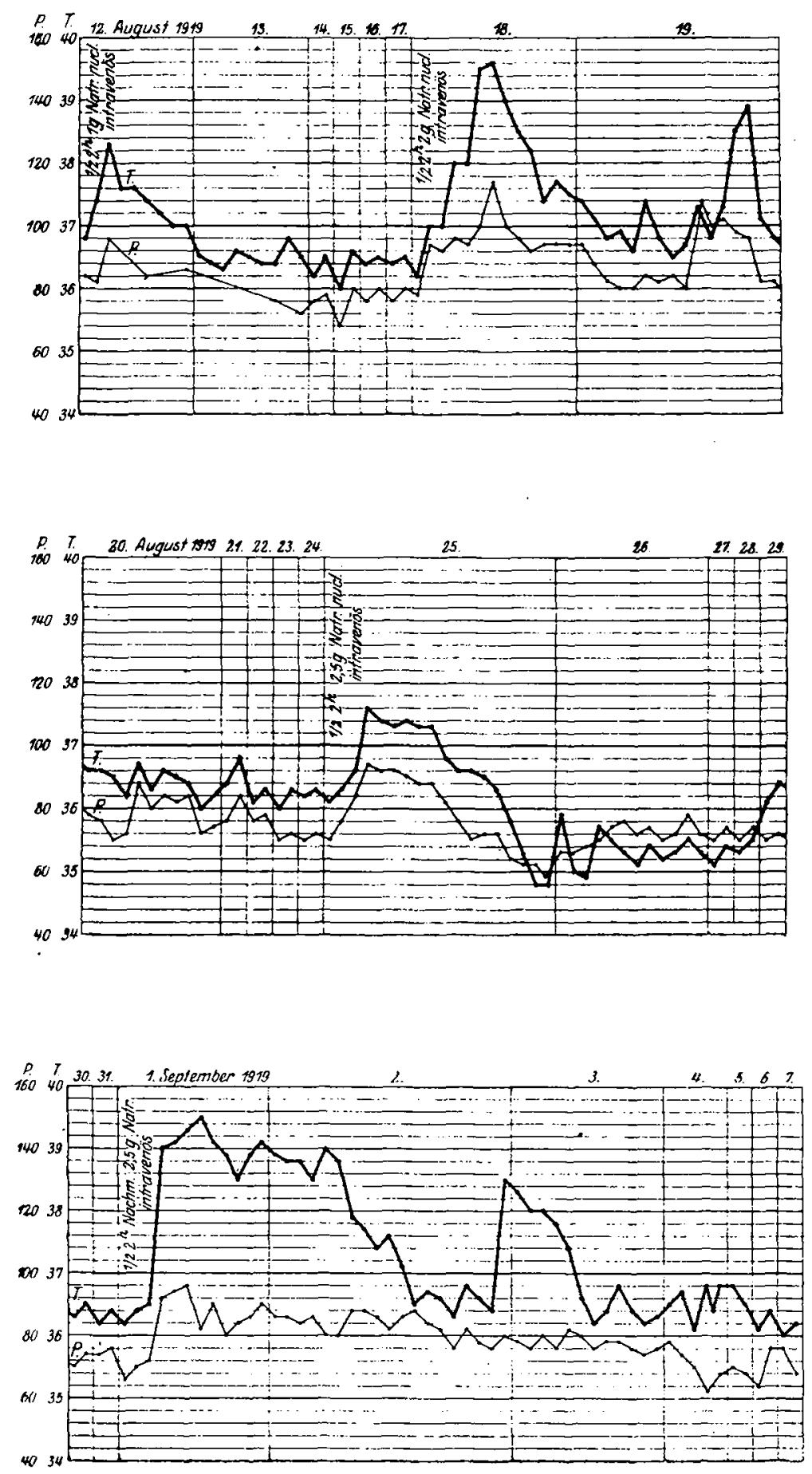
hurve 4. (Se.)
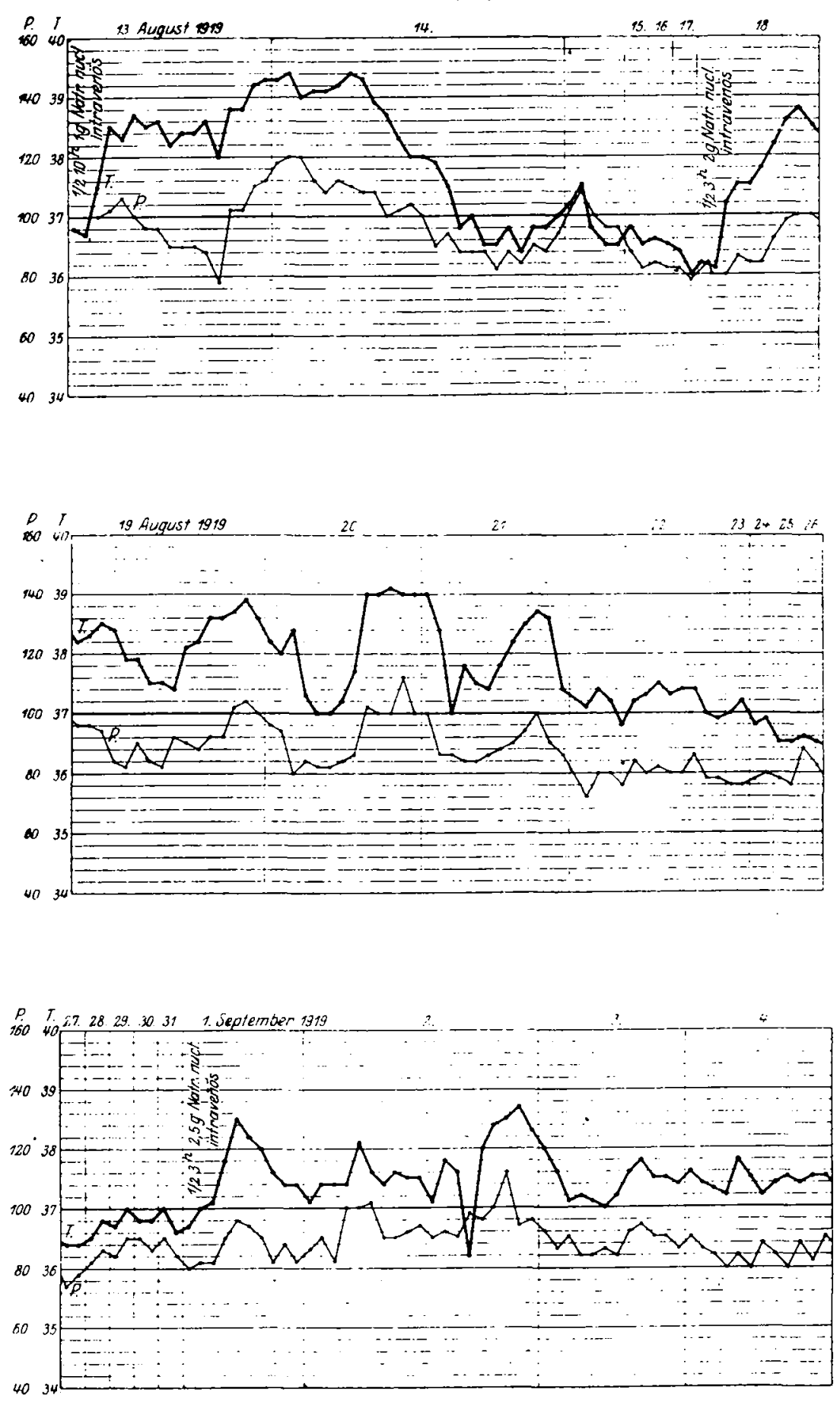
Fortsetzung von Kurve 4. (Se.)
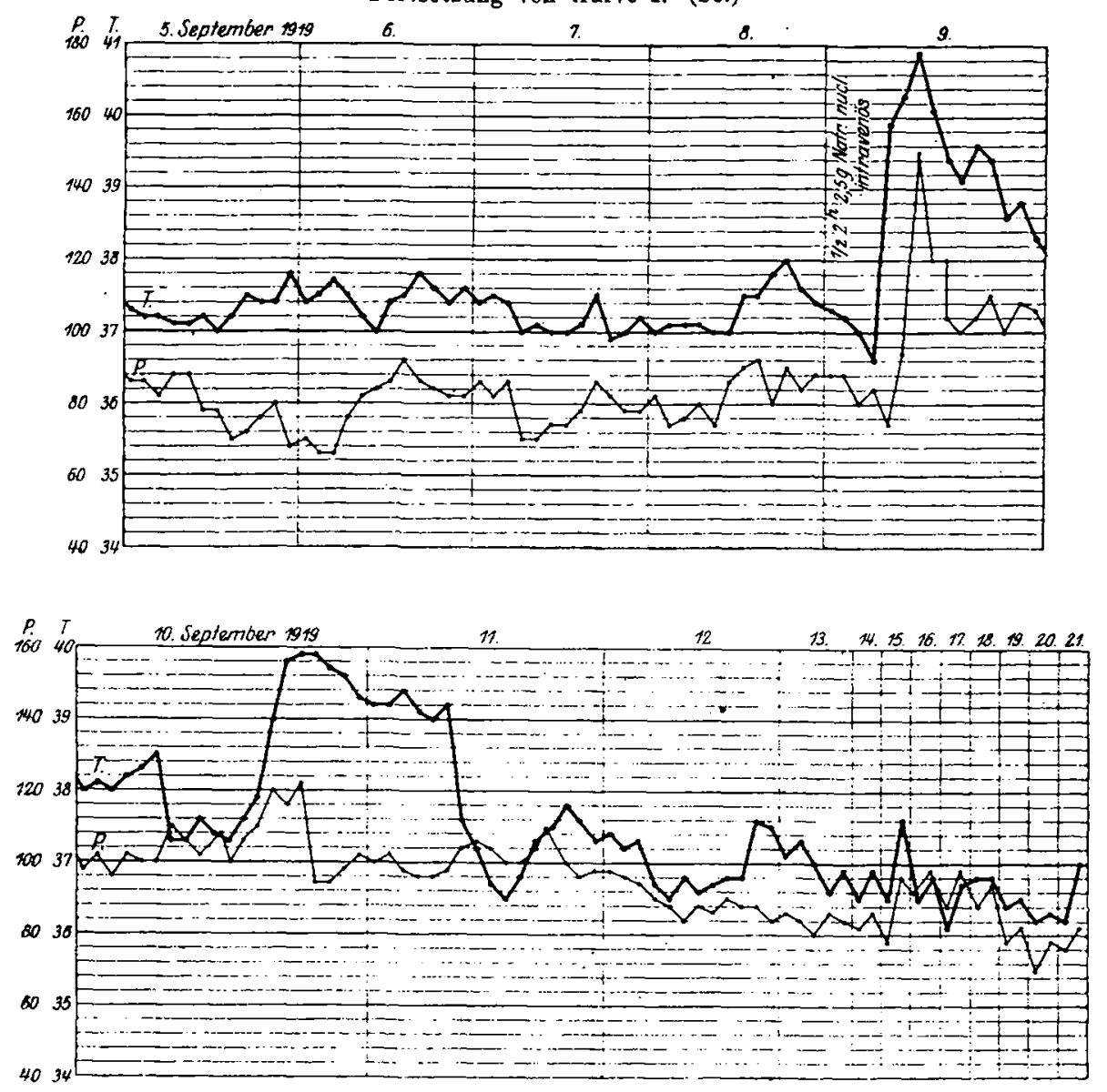

zeigten, in der im J'ierkörper nach parenteraler Zufuhr eiweissartiger Substanzen anaphylaktische Vorgänge in Erscheinung zu treten pflegen. Die Ueberempindlichkeit hat sich allerdings bei unseren Fällen niemals durch den typischen Shock manifestiert. sondern lediglich durch die Lebhaftigkeit der Reaktion. Ausserdem kann ron anaphylaktischen Vorgängen in unseren Fällen kaum die Rede sein, da die Fähigkeit, zu sensibilisieren, ja an das Eiweissmolekül gebunden ist. Eine erworbene Ueberempfindlichkeit gegen nicht eiweissartige Substanzen ist ja z. B. von Adnecco nach wiederholten Kokain- und ron Richet nach wiederholten Apomorphininjektionen beschrieben worden ${ }^{1}$ ).

Es fragt sich aber, ob die von mir beobachtete Emplindlichkeit des Körpers gegen wiederholte Injektionen von Nukleinsäure nicht auf dem Vorhandensein von Eiweissresten beruht, die dem Mittel bei seinem Entstehungsmodus anhaften könnten. So erklärt sich nach Seligmann z. B. das sensibilisierende Vermögen gewisser roher Fette und Oele, von

1) Vgl.E. Soligmann, "Anaphylaxio", Handbuoh d. Biochomie von Opponhoimer. Fischer 1913 . 
aus Hühnereigelb gewonnenen Lipoidextrakten aus spuren ron lijweissresten, die diesen Stoffen dank des Milieus, aus dem sic stammen, anhaften ${ }^{1}$ ). Auch die sensibilisierende Fähigkeit bestimmter Gifte, z. B. des Kobragiftes, der Kongestine und der Phytalbumine führt er auf den liweissgehalt dieser Stoffe zurück.

Die Erfolglosigkeit der Nukleinsäuretherapie bei progressiver Paralyse, die vielleicht darauf beruhte, dass wir meistens nicht ganz frische Fälle behandelten: drïngte nach Klärung. Befriedigenden Iufschluss gab mir ein Fall, der während der Behandlung nach wiederholter Applikation des Vittels ad exitum und zur Obduktion kam.

Der pathologisch-anatomische Befund, bei dessen Deutung mich Fräulein Dr. Krieger Pathologisch-anatomisches Institut: Prof. Koissle) gültig unterstützte. wies iul cine gesteigerte Lebhaftigkcit des hämatopoetischen Systems. besonders ron Mil\% und Knochenmark hin. Es ergaben sich ganz ahnliche Resultate. wie sie seiner Zeit von Parlavecchio beschrieben worden sind ${ }^{3}$. Auffallend war: dass dic Leber geradezu von Leukozyten überschwemmt war, während sich $1 \mathrm{~m}$ Gehirn eher eine Verminderung der dort durchschnittlich angetroffenen Leukozytenzahl fand.

Nachdem unsere therapeutischen Versuche mit Nukleinsäure längst abgeschlossen waren. stiess ich auf cine Arbeit ron Tschernoruzki aus dem Laboratorium für biologische Chemie des Instituts für experimentelle Medizin zu St. Petersburg: „Ueber die gegenseitige Wirkung vou Tukleinsäurc und nuklcinspaltendem Ferment im tierischen Organismus ${ }^{4}$ ), die auf die weitgehende $\Lambda$ nalogie von Sukleinsäure und Infektion auf den tierischen Organismus und der durch das Nukleaseferment bedingten nukleolytischen Funktion des Körpers bei Infektionen hinweist.

${ }_{n}$ Der Nuklease muss-. so meint der Verfasser. ${ }^{-}$wie mir scheint. im Kampf des Organismus mit Infektion und ,lebenden Zellen' cine besonders grosse Bedeutung zukommen, da sowohl Bakterienleib, wie tierische Zelle zu 50-75 pCt. aus Nukleoproteiden bestehen." Vergleichende Organuntersuchungen auf den Gehalt an Nuklease nach Nukleinsäureinjektionen ergaben aber. dass im Gehirn eher eine yuantitative Herabsetzung dieses Ferments, in der Leber dagegen eine Vermehrung desselben festzustcllen war. Da nun die Jeukozyten unter allen Zellen des Organismus am reichsten an Nuklease sind, so stimmte die Feststellung 'T'schernoruzki's sehr gut mit unseren anatomischen Untersuchungen überein und sprach einer wirklich kausalen, gegen die im Grosshirn ansässigen Spirochäten gerichteten Therapic das Urteil.

Dass durch die durch Nukleinsäure hervorgerufene Hyperleukozytose des Blutes eine Elimination toxischer Stoffwechselprodukte, die als Zer-

1) Seligmann, S. $26 \pi^{\circ}$.

2) Eine grössere Serie ron Fällen wurde auf der Mänverabteilung von Atteilungsarzt Dr. Densow bebandelt.

3) Arch, f. klin. Chir, Bit. 3(1. S. 2) 2).

4) Biocbem. Leitschr. 191:. Bd. 44. S. $35 \%$. 
fallsprodukte des Nervensystems in den Kreislauf,gelangen, erzielt werden kann, erscheint mir durchaus möglich, und dass hierdurch vorübergehende Besserungen im Krankheitsbild erreicht werden können, liegt theoretisch ebenso im Rahmen der Möglichkeit. Beim jetzigen Stand des Paralyseproblems befriedigt uns aber eine derartige symptomatische Therapie nicht mehr, weil es uns Bedürfnis ist, direkt auf die in der Grosshirnrinde vorhandenen Spirochätennester einzuwirken.

Die Nukleinsäureinjektionen wurden nicht immer ohne Zwischenfälle vertragen. Stärkere Fieberreaktionen wurden fast regelmässig durch Schültelfrosi eingeleitet und gingen zuweilen mit Durchfällen einher. Erscheinungen von seiten der Niere zeigten sich nur im Falle Se. (vgl. Kurve 4). Hier stellten sich nach der vierten Injektion nach 2,5 g Natrium nucleinicum ganz äholiche Vergiftungserscheinungen ein, wie sie von Tschernoruzki beim Hunde nach intravenöser Darreichung von $18,45 \mathrm{~g}(1,5 \mathrm{~g}$ pro Kilogramm Körpergewicht) beschrieben worden sind: A pathie; Schwäche, Durchfall und Erbrechen. Ausserdem wurde ein schwerer Anfall von Herzschwäche beobachtet, der durch reichliche Herzmittel überwunden wurde. Im Harn, der anfangs stark vermindert war und hohes spezifisches Gewicht zeigte, fand sich Eiweiss $(1 / 4 \mathrm{pM}$.); mikroskopisch wurden zahlreiche hyaline und granulierte Zylinder, reichliche leukozyten und Liythrozyten gefunden. Oedeme fehlten ganz. Es besteht kein 7weifel, dass die Patientin damals das Bild einer hämorrhagischen Nephritis bot, wie es ja bei Vergiftungen sich einstellt. Gliicklicherweise hatte die Kranke nach 3 Wochen die Schidigung rollstïndig überwunden.

In diesem Zusammenhang verdient die Tatsache Erwähnung, dass Ewald ${ }^{1}$ ) einen Versuchshund nach wiederholter intravenöser Darreichung von je $5 \mathrm{~g}$ Hefe- und Thymusnukleinsäure an hämorrhagischer Nephritis verlor. Ich stehe auf Grund meiner Erfahrungen der Annahme des Autors, diese nicht auf Kosten der Injektionen zu setzen, sehr skeptisch gegenüber.

Wir gewannen aus unserer Versuchsreihe die Veberzeugung, dass man bei intravenösen Injektionen von Natrium nucleinicum in höheren Dosen mit lebensgefährlichen Zwischenfällen rechnen muss. Dies veranlasste mich, die nach dieser Richtung unternommenen therapeutischen Versuche abzubrechen. Theoretisch erscheinen sie uns aus mancherlei Gründen der Beachtung wert.

1) Zeitschr. f. exper. Path. u. Ther. Bd. 12. 